\title{
On the impact of boundary conditions on dual consistent finite difference discretizations
}

\author{
Jens Berg and Jan Nordström
}

\section{Linköping University Post Print}

\section{N.B.: When citing this work, cite the original article.}

Original Publication:

Jens Berg and Jan Nordström, On the impact of boundary conditions on dual consistent finite difference discretizations, 2013, Journal of Computational Physics, (236), , 41-55.

http://dx.doi.org/10.1016/j.jcp.2012.11.019

Copyright: Elsevier

http://www.elsevier.com/

Postprint available at: Linköping University Electronic Press

http://urn.kb.se/resolve?urn=urn:nbn:se:liu:diva-86397 


\title{
On the impact of boundary conditions on dual consistent finite difference discretizations
}

\author{
Jens Berg* \\ Uppsala University, Department of Information Technology, SE-751 05, Uppsala, Sweden \\ Jan Nordström \\ Linköping University, Department of Mathematics, SE-581 83, Linköping, Sweden
}

\begin{abstract}
In this paper we derive well-posed boundary conditions for a linear incompletely parabolic system of equations, which can be viewed as a model problem for the compressible Navier-Stokes equations. We show a general procedure for the construction of the boundary conditions such that both the primal and dual equations are wellposed. The form of the boundary conditions is chosen such that reduction to first order form with its complications can be avoided.

The primal equation is discretized using finite difference operators on summationby-parts form with weak boundary conditions. It is shown that the discretization can be made energy stable, and that energy stability is sufficient for dual consistency. Since reduction to first order form can be avoided, the discretization is significantly simpler compared to a discretization using Dirichlet boundary conditions.

We compare the new boundary conditions with standard Dirichlet boundary conditions in terms of rate of convergence, errors and discrete spectra. It is shown that the scheme with the new boundary conditions is not only far simpler, but also has smaller errors, error bounded properties, and highly optimizable eigenvalues, while maintaining all desirable properties of a dual consistent discretization.
\end{abstract}

Keywords: High order finite differences; Summation-by-parts; Superconvergence;

${ }^{*}$ Corresponding author: Jens Berg

Address: Division of Scientific Computing, Department of Information Technology, Uppsala University, Box 337, SE-751 05, Uppsala, Sweden

Phone: +46 $18-4716253$

Fax: +4618 523049, +4618511925

E-mail: jens.berg@it.uu.se

Preprint submitted to Elsevier

November 30, 2012 
Boundary conditions; Dual consistency; Stability

\section{Introduction}

Functionals can represent the lift or drag on an aircraft, energy or any other scalar quantity computed from the solution to a partial differential equation (PDE). In many engineering applications, high order accurate functionals are often of greater interest than accurate solutions of the equations themselves. Whenever there is a functional involved, the concept of duality becomes important. The solution of a PDE resides in some function space, and the set of all bounded linear functionals on that space is called its dual space. Knowledge of the functional of interest can thus be obtained by studying the dual space. This is the main topic in functional analysis and references can be found in any standard textbook.

The dual equations need, as the primal equations, to be supplied with the correct boundary conditions in order for a solution to exist and be unique. Like the dual differential operator, the dual boundary conditions depend on the primal problem and it is a non-trivial task to construct boundary conditions such that both the primal and dual problems are well-posed. The most common choice is Dirichlet boundary conditions since the analysis of the continuous equations is simplified. However, it is well-known that Dirichlet boundary conditions cause large reflections and reduces the accuracy and stability properties of a numerical scheme [18]. Other kinds of boundary conditions are beneficial for a discretization, but complicate the analysis

of the dual equations. Here, we shall study boundary conditions of far-field type which have been shown to be beneficial for discretizations [19, 23, 21].

In numerical analysis, and in particular for computational fluid dynamics problems, duality has been exploited for optimal control problems $[27,15,8]$, error estimation $[26,34,35,12,7]$ and convergence acceleration $[9,25,13,4]$. An extensive summary of the use of adjoint problems can be found in [10], and more recently in [6] with focus on error estimation and adaptive mesh refinement.

In this paper we will use a finite difference method on summation by parts (SBP) form with boundary conditions weakly imposed by the simultaneous approximation term (SAT). There has recently been a development of the quadrature properties of SBP-SAT discretizations. The base for an SBP operator is a norm matrix, denoted by $P$. The norm matrix is an integration operator for equidistant grid points. The integration properties of $P$ has been studied in [14] and it was shown that $P$ is a high-order accurate quadrature rule which extends the Gregory formulas. In [13], the discretization of steady problems were considered. The authors showed that certain SBP-SAT discretizations, so called dual consistent discretizations, led to 
superconvergent functionals if the same $P$ was used in the discretization as in the functional evaluation. The theory of dual consistency and superconvergence was extended to time-dependent problems in [4]. Several problems were analyzed and it was shown that dual consistency and stability implies superconvergence of linear functionals.

In order to avoid additional theoretical difficulties in [4], Dirichlet boundary conditions for both the primal and dual problems were used. The Dirichlet boundary conditions ensured that both problems were well-posed without additional efforts. In an Euler or Navier-Stokes calculation, however, Dirichlet boundary conditions are rarely used at far-field boundaries. Unless exact boundary data is known, Dirichlet boundary conditions cause reflections which pollute the solution.

In this paper we will investigate the potential gain, or loss, when replacing the Dirichlet boundary conditions with more sophisticated ones. The aim of the paper is to derive well-posed boundary conditions for both the primal and dual problems such that the complications of having Dirichlet boundary conditions are removed, while maintaining all desirable properties of a dual consistent discretization and sophisticated boundary conditions.

\section{Preliminaries}

In this paper, we will consider time-dependent partial differential equations of the form

$$
\begin{aligned}
u_{t}+L(u) & =f, \\
J(u) & =(g, u),
\end{aligned}
$$

where $J(u)$ is a linear functional output of interest with $g=g(x, t)$ being an arbitrary weight function. $L$ can be either linear or non-linear and $u$ can represent either a scalar or vector valued function. Detailed descriptions can be found in [4] but for convenience, we summarize the main preliminaries here.

The inner product is the standard $L^{2}$-inner product

$$
(u, v)=\int_{\Omega} u^{T} v d \Omega .
$$

In [4], the concept of spatial dual consistency was introduced to avoid treating the full time-dependent dual equations when discretizing using a method of lines. The concept is motivated by the following. To find the dual problem, we follow the 
notation in $[4,13]$, and seek a function $\theta$ in some appropriate function space, such that

$$
\int_{0}^{T} J(u) d t=\int_{0}^{T}(\theta, f) d t .
$$

A formal computation (assume $L$ linear and $u, \theta$ to have compact support in space) gives

$$
\begin{aligned}
\int_{0}^{T} J(u) d t & =\int_{0}^{T} J(u) d t-\int_{0}^{T}\left(\theta, u_{t}+L u-f\right) d t \\
& =\int_{0}^{T}\left(\theta_{t}-L^{*} \theta+g, u\right) d t-\int_{\Omega}[\theta u]_{0}^{T} d \Omega+\int_{0}^{T}(\theta, f) d t,
\end{aligned}
$$

where $L^{*}$ is the formal adjoint, or dual, operator associated with $L$ under the inner product such that $(\theta, L u)=\left(L^{*} \theta, u\right)$. By having homogeneous initial conditions for the primal problem, we obtain the time-dependent dual problem as

$$
-\theta_{t}+L^{*} \theta=g
$$

where we have to put an initial condition for the dual problem at time $t=T$. The time transformation $\tau=T-t$ transforms (2) to

$$
\theta_{\tau}+L^{*} \theta=g
$$

with an initial condition at $\tau=0$. A discretization which simultaneously approximates the spatial primal and dual operator consistently, is called spatially dual consistent and produces superconvergent time-dependent linear functionals if the scheme for the primal problem is stable [4].

A difference operator for the first derivative is said to be on SBP form if it can be written as $D_{1}=P^{-1} Q$. $P$ defines a norm by $\left\|u_{h}\right\|^{2}=u_{h}^{T} P u_{h}$ and $Q$ satisfies the SBP property $Q+Q^{T}=E_{N}-E_{0}$, where

$$
E_{N}=\operatorname{diag}[0, \ldots, 0,1], \quad E_{0}=\operatorname{diag}[1,0, \ldots, 0] .
$$

The second derivative operator can be constructed either by applying the first derivative twice, i.e. $D_{2}=\left(P^{-1} Q\right)^{2}$ which results in a wide operator, or a compact operator with minimal bandwidth of the form

$$
D_{2}=P^{-1}\left(-H+\left(E_{N}-E_{0}\right) S\right)
$$


as described in $[5,17,16]$. In this paper, we consider only diagonal $[28]$ norms and wide second derivative operators. The diagonal norm is flexible for realistic simulations as the resulting schemes can be shown to be energy stable under curvilinear coordinate transforms. This does not hold for non-diagonal norms [20, 22, 30, 32].

A first derivative SBP operator is essentially a $2 s$-order accurate central finite difference operator which has been modified close to the boundaries such that it becomes one-sided. Together with the diagonal norm, the boundary closure is accurate of order $s$ making the SBP operator accurate of order $s+1$ in general [28]. For problems with second derivatives, the compact operator can be modified with higher order accurate boundary closures to gain one extra order of accuracy $[17,33]$.

A discretization of the primal problem (1) can be written as

$$
\frac{d}{d t} u_{h}+L_{h} u_{h}=f,
$$

where $u_{h}$ is the discrete approximation of $u$ and $L_{h}$ is a discrete approximation of $L$, including the boundary conditions. The discrete inner product in an SBP setting is defined by

$$
\left(u_{h}, v_{h}\right)_{h}=u_{h}^{T} P v_{h}
$$

and hence the discrete adjoint operator can be computed, according to the definition

$$
\left(v_{h}, L_{h} u_{h}\right)_{h}=\left(L_{h}^{*} v_{h}, u_{h}\right)_{h},
$$

as

$$
L_{h}^{*}=P^{-1} L_{h}^{T} P .
$$

The proof that a stable and spatially dual consistent SBP scheme produces superconvergent linear functionals is presented in [4]. The proof is based on the fact that the mass matrix, $P$, in the norm is a $2 s$-order accurate integration operator $[14,13]$.

The procedure for constructing stable schemes which produce superconvergent linear functionals can now be summarized as follows;

1. Determine boundary conditions such that both the primal and dual problems are well-posed

2. Discretize the primal problem and ensure stability

3. Compute $L_{h}^{*}$ and choose the remaining parameters (if any) such that the continuous adjoint $L^{*}$ is consistently approximated together with the dual boundary conditions

Note that a stable and consistent discretization of the primal problem does not imply that the dual problem is consistently approximated. 


\section{Linear incompletely parabolic system}

We shall study the linear incompletely parabolic system of equations on $0 \leq x \leq 1$ given by

$$
U_{t}+A U_{x}=B U_{x x}
$$

where $U=[p, u]^{T}$ and

$$
A=\left[\begin{array}{cc}
\bar{u} & \bar{c} \\
\bar{c} & \bar{u}
\end{array}\right], \quad B=\left[\begin{array}{ll}
0 & 0 \\
0 & \varepsilon
\end{array}\right],
$$

together with a linear functional of interest,

$$
J(U)=\int_{0}^{1} G^{T} U d x .
$$

Equation (3) can be viewed as the symmetrized [2], one-dimensional Navier-Stokes equations, linearized around a flow field with constant velocity $\bar{u}>0$ and speed of sound $\bar{c}>0$. In this case, we assume $\bar{u}<\bar{c}$ and it can be shown [24, 29] that (3) requires two boundary conditions at the inflow boundary, $x=0$, and one at the outflow boundary at $x=1$, in both the subsonic and supersonic case. Even though this is a model problem, we denote the case $\bar{u}<\bar{c}$ as subsonic, and supersonic if $\bar{u}>\bar{c}$.

\subsection{Dirichlet boundary conditions}

In [4], equation (3) was supplied with the Dirichlet boundary conditions

$$
p(0, t)=0, \quad u(0, t)=0, \quad u(1, t)=0 .
$$

An energy estimate results in

$$
\|U\|_{t}^{2}+\left\|B U_{x}\right\|^{2} \leq 0
$$

where we used the notation $\|U\|_{t}^{2}=\frac{d}{d t}\left(\|U\|^{2}\right)$. Note that the boundary conditions cancel all boundary terms completely, and does not give any additional damping of the energy, $\|U\|^{2}$.

The spatial dual operator was obtained by reducing (3) to a first order system by introducing the auxiliary variable $v=\sqrt{\varepsilon} u_{x}$. There are several drawbacks with this technique. The most obvious one is that it results in a larger system of equations which complicates the analysis. The drawbacks of the first order form also carries over to the discretization. In the discretization of the first order form, there are nine unknown penalty parameters in the SAT procedure which have to be determined for stability and dual consistency [4]. This makes extensions to larger system in multiple dimensions complicated. 


\subsection{Flux based boundary conditions}

The new boundary conditions we consider are of the form

$$
H_{L, R} U \mp B U_{x}=G_{L, R},
$$

where $H_{L, R}$ will be determined for well-posedness of both the primal and dual problems.

There are many different forms of the matrices $H_{L, R}$ in (6) which give wellposed inflow or outflow boundary conditions. The typical way to determine the structure of $H_{L, R}$ is to diagonalize the hyperbolic part of the equation and consider the ingoing or outgoing characteristics. This method will provide an energy estimate with optimal damping properties [19]. However, the dual problem associated with the linear functional (4) will most likely be ill-posed. Well-posedness of the primal problem does not imply well-posedness of the dual problem.

Since we are only interested in the spatial dual operator, it is sufficient to consider the steady, inhomogeneous, problem

$$
A U_{x}-B U_{x x}=F .
$$

In this case, the differential operator $L$ is given by

$$
L=A \frac{\partial}{\partial x}-B \frac{\partial^{2}}{\partial x^{2}}
$$

and we seek $\theta=[\phi, \psi]^{T}$ such that $J(U)=(\theta, F)$. Integration by parts gives

$$
\begin{aligned}
J(U) & =(G, U)-(\theta, L U-F) \\
& =\left(G-L^{*} \theta, U\right)-\left[\theta^{T} A U-\theta^{T} B U_{x}+\theta_{x}^{T} B U\right]_{0}^{1}+(\theta, F),
\end{aligned}
$$

where $L^{*} \theta=-A \theta_{x}-B \theta_{x x}$ and hence the dual operator is given by

$$
L^{*}=-A \frac{\partial}{\partial x}-B \frac{\partial^{2}}{\partial x^{2}} \text {. }
$$

To determine the boundary conditions for the dual problem, we have to find a minimal set of conditions such that

$$
\left[\theta^{T} A U-\theta^{T} B U_{x}+\theta_{x}^{T} B U\right]_{0}^{1}=0
$$

after the homogeneous boundary conditions for the primal problem have been applied. This is what put restrictions on the matrices $H_{L, R}$ in (6). Not only does the boundary terms have to vanish, they will also have to satisfy the correct number and form for the dual equation. Wrong choice of boundary conditions for the primal problem will cause the dual problem to be ill-posed [24, 29]. 


\subsubsection{Left boundary conditions}

To simplify the analysis, we assume the left boundary condition to be homogeneous,

$$
H_{L} U-B U_{x}=0 .
$$

By considering only the terms at $x=0$, we can write (8) as

$$
\theta^{T}\left(A U-B U_{x}\right)+\theta_{x}^{T} B U=U^{T}\left(\left(A-H_{L}^{T}\right) \theta+B \theta_{x}\right)
$$

after having applied the homogeneous boundary conditions (9) for the primal equation. The boundary conditions for the dual equation are thus given by

$$
\left(A-H_{L}^{T}\right) \theta+B \theta_{x}=0 .
$$

The form of the matrix $H_{L}$ can now be determined. Since the left boundary is an inflow boundary for the primal equation, but an outflow boundary for the dual equation, only one boundary condition is allowed for the dual equation. The boundary conditions (10) hence have to be of rank one and thus it is required that

$$
A-H_{L}^{T}=\left[\begin{array}{cc}
0 & 0 \\
\alpha_{L} & \beta_{L}
\end{array}\right]
$$

or equivalently

$$
H_{L}=\left[\begin{array}{cc}
\bar{u} & \bar{c}-\alpha_{L} \\
\bar{c} & \bar{u}-\beta_{L}
\end{array}\right] .
$$

Any other form of $H_{L}$ would impose too many boundary conditions for the dual equation and it would not be well-posed. The coefficients $\alpha_{L}, \beta_{L}$ have to be chosen such that we obtain an energy estimate for both the time-dependent primal and dual problems.

We can now turn our attention back to the primal equation. The primal equation needs two boundary conditions at $x=0$, and hence $H_{L}$ is required to have a non-zero top row. This requirement is automatically fulfilled since $\bar{u}>0$ by assumption. To determine the coefficients $\alpha_{L}, \beta_{L}$ we apply the energy method to (3) and consider only the left boundary terms. We get

$$
\|U\|_{t}^{2}=U^{T} A U-U^{T} B U_{x}-U_{x}^{T} B U
$$

By applying the homogeneous boundary conditions (9), we can write (13) as

$$
\|U\|_{t}^{2}=-U^{T} M_{L} U
$$


where

$$
M_{L}=-A+H_{L}+H_{L}^{T}=\left[\begin{array}{cc}
\bar{u} & \bar{c}-\alpha_{L} \\
\bar{c}-\alpha_{L} & \bar{u}-2 \beta_{L}
\end{array}\right]
$$

and we have to choose the coefficients $\alpha_{L}, \beta_{L}$ such that $M_{L} \geq 0$. There are several strategies for how to choose the parameters $\alpha_{L}$ and $\beta_{L}$ such that $M_{L} \geq 0$. The most general is to compute the eigenvalues of $M_{L}$ and determine the parameters such that all eigenvalues are positive. In this simple $2 \times 2$ case, the eigenvalues can be directly computed as

$$
\mu_{1,2}^{(L)}=\bar{u}-\beta_{L} \pm \sqrt{\left(\bar{u}-\beta_{L}\right)^{2}-\bar{u}\left(\bar{u}-2 \beta_{L}\right)+\left(\bar{c}-\alpha_{L}\right)^{2}}
$$

and it is required that

$$
\left(\bar{c}-\alpha_{L}\right)^{2}-\bar{u}\left(\bar{u}-2 \beta_{L}\right) \leq 0 .
$$

For larger systems and more complicated equations, this approach might not be possible as the eigenvalues are not analytically available. A simpler strategy is proposed in

Proposition 3.1. The primal equation (3) is well-posed with the left boundary conditions given in (9), where $H_{L}$ is defined in (12) and the parameters $\alpha_{L}, \beta_{L}$ satisfy

$$
\alpha_{L}=\bar{c}, \quad \beta_{L} \leq \frac{\bar{u}}{2}
$$

Proof. The primal problem requires two boundary conditions at $x=0$. Hence the top row of $H_{L}$ needs to be non-zero. We can see from (12) that this is always the case since $\bar{u}>0$ by assumption. By inserting the values in (15) into (14), we get

$$
M_{L}=\left[\begin{array}{cc}
\bar{u} & 0 \\
0 & \bar{u}-2 \beta_{L}
\end{array}\right]
$$

which is diagonal with non-negative diagonal entries.

Note that the strategy in proposition 3.1 is to i) cancel the off-diagonal terms and ii) ensure that the remaining diagonal terms have the correct sign.

A third option is to determine $\alpha_{L}$ and $\beta_{L}$ such that the boundary conditions converge uniformly to a well-posed set of boundary conditions for the hyperbolic system

$$
U_{t}+A U_{x}=0
$$

as $\varepsilon \rightarrow 0$. The third option will be discussed later, and for now we consider the choices in (15). 
It is not only the primal equation which has to be well-posed. The time-dependent dual equation with its dual boundary conditions need also be well-posed with the conditions given in (15). By introducing the time transformation $\tau=T-t$, we can write the time-dependent dual equation as

$$
\theta_{\tau}-A \theta_{x}=B \theta_{x x}
$$

The well-posedness of the dual problem is proven in

Proposition 3.2. The time-dependent dual problem (16) is well-posed with the dual boundary conditions (10) where $H_{L}$ is defined in (12) with the parameters given in (15).

Proof. The dual problem is only allowed to have one boundary condition at $x=0$. It is thus required that the top row of $A-H_{L}^{T}$ is zero. By construction of $H_{L}$, this is the case as can be seen in (11). By applying the energy method to (16), and only considering the terms at $x=0$, we obtain

$$
\begin{aligned}
\|\theta\|_{\tau}^{2} & =-\theta^{T} A \theta-\theta^{T} B \theta_{x}-\theta_{x}^{T} B \theta \\
& =-\theta^{T}\left(-A+H_{L}+H_{L}^{T}\right) \theta \\
& =-\theta^{T} M_{L} \theta
\end{aligned}
$$

after applying the boundary conditions (10). The semi-definiteness of $M_{L}$, with the choices (15), were already proven in the energy estimate of the primal equation.

To summarize, the left homogeneous boundary conditions for the primal problem are given in (9) and for the dual problem in (10), where $H_{L}$ is defined in (12) with the coefficients given in (15).

\subsubsection{Right boundary conditions}

The right boundary, $x=1$, is an outflow boundary for the primal problem and hence only one boundary condition can be used, while we have two variables in the system. This immediately puts restrictions on the homogeneous primal boundary condition

$$
H_{R} U+B U_{x}=0
$$

in such a way that $H_{R}$ is required to have the form

$$
H_{R}=\left[\begin{array}{cc}
0 & 0 \\
\alpha_{R} & \beta_{R}
\end{array}\right] \text {. }
$$


For any other form of $H_{R}$, too many boundary conditions are placed at the outflow boundary and the primal problem is ill-posed. The coefficients $\alpha_{R}, \beta_{R}$ have to be determined such that both the time-dependent primal and dual problems are wellposed.

Once the form of $H_{R}$ in (18) has been determined, we must make sure that the correct number of boundary conditions are imposed on the dual problem. To determine the boundary conditions for the dual problem, we restrict (8) to the terms at $x=1$. After applying the homogeneous primal boundary conditions (17) we obtain

$$
\theta^{T}\left(A U-B U_{x}\right)+\theta_{x}^{T} B U=U^{T}\left(\left(A+H_{R}^{T}\right) \theta+B \theta_{x}\right)
$$

and hence the boundary conditions for the dual problem are given by

$$
\left(A+H_{R}^{T}\right) \theta+B \theta_{x}=0
$$

where

$$
A+H_{R}^{T}=\left[\begin{array}{cc}
\bar{u} & \bar{c}+\alpha_{R} \\
\bar{c} & \bar{u}+\beta_{R}
\end{array}\right] .
$$

Since the dual problem requires two boundary conditions at $x=1$, it is required that the top row of $A+H_{R}^{T}$ is non-zero. We can see that this requirement is automatically fulfilled since $\bar{u}>0$ by assumption.

The coefficients $\alpha_{R}$ and $\beta_{R}$ can now be determined such that we obtain energy estimates for both the primal and dual equations. The energy method applied to the time-dependent dual problem (16), with the homogeneous dual boundary conditions in (19), gives

$$
\|\theta\|_{\tau}^{2}=-\theta^{T} M_{R} \theta
$$

where

$$
M_{R}=A+H_{R}+H_{R}^{T}=\left[\begin{array}{cc}
\bar{u} & \bar{c}+\alpha_{R} \\
\bar{c}+\alpha_{R} & \bar{u}+2 \beta_{R}
\end{array}\right] .
$$

As this is a $2 \times 2$ system, we can directly compute the eigenvalues of the symmetric matrix $M_{R}$ as

$$
\mu_{1,2}^{(R)}=\bar{u}+\beta_{R} \pm \sqrt{\left(\bar{u}+\beta_{R}\right)^{2}-\bar{u}\left(\bar{u}+2 \beta_{R}\right)+\left(\bar{c}+\alpha_{R}\right)^{2}}
$$

and see that they are both non-negative if we choose $\alpha_{R}$ and $\beta_{R}$ such that

$$
\left(\bar{c}+\alpha_{R}\right)^{2}-\bar{u}\left(\bar{u}+2 \beta_{R}\right) \leq 0 .
$$

Again, in more realistic situations the eigenvalues might not be analytically computable and we use the same strategy as before - to cancel the off-diagonal elements and ensure that the remaining diagonal terms have the correct sign. The values of $\alpha_{R}$ and $\beta_{R}$ with this strategy are given in 
Proposition 3.3. The time-dependent dual problem (16) is well-posed with the right dual boundary conditions in (19) where the parameters in $H_{R}$ satisfy

$$
\alpha_{R}=-\bar{c}, \quad \beta_{R} \geq-\frac{\bar{u}}{2}
$$

Proof. The dual problem requires two boundary conditions at $x=1$ and hence the top row of $A+H_{R}^{T}$ must be non-zero. That this is always the case can be seen in (20) since $\bar{u}>0$ by assumption. By substituting the relations in (22) into (21), we obtain

$$
M_{R}=\left[\begin{array}{cc}
\bar{u} & 0 \\
0 & \bar{u}+2 \beta
\end{array}\right]
$$

which is diagonal with non-negative diagonal entries.

The well-posedness of the primal problem is given in

Proposition 3.4. The primal problem (3) is well-posed with the right boundary conditions in (17), where the parameters in $H_{R}$ are given in (22).

Proof. The primal problem requires one boundary condition at $x=1$ which is fulfilled by the construction of $H_{R}$ in (18). As before, the energy method applied to the timedependent primal problem gives

$$
\begin{aligned}
\|U\|_{t}^{2} & =-U^{T}\left(A+H_{R}+H_{R}^{T}\right) U \\
& =-U^{T} M_{R} U
\end{aligned}
$$

where semi-definiteness of $M_{R}$ is already proven from the energy estimate of the dual equation.

To summarize, the right homogeneous boundary conditions for the primal problem are given in (17) and for the dual problem in (19), where $H_{R}$ is defined in (18) with the coefficients given in (22).

Remark 3.1. Note how it is the problem which requires the least number of boundary conditions which sets restrictions on the form of the boundary conditions. When also considering well-posedness of the dual problem, it can be used to reduce the number of unknown parameters in the boundary conditions of the primal problem.

Remark 3.2. The energy estimate, when considering all terms, for the primal problem is given by

$$
\|U\|_{t}^{2}+\left\|B U_{x}\right\|^{2}=-U^{T} M_{L} U-U^{T} M_{R} U
$$


and for the dual problem by

$$
\|\theta\|_{\tau}^{2}+\left\|B \theta_{x}\right\|^{2}=-\theta^{T} M_{L} \theta-\theta^{T} M_{R} \theta .
$$

Both matrices $M_{L}$ and $M_{R}$ have at least one positive eigenvalue and hence the boundary conditions contribute to damping of the energy. In the energy estimate (5) with Dirichlet boundary conditions, no additional damping is obtained.

\subsection{Convergence to the hyperbolic system}

As was discussed previously, the parameters $\alpha_{L, R}$ and $\beta_{L, R}$ can be chosen such that they converge to well-posed boundary conditions of the hyperbolic system

$$
U_{t}+A U_{x}=0
$$

as $\varepsilon \rightarrow 0$. The energy method applied to (23) results in

$$
\|U\|_{t}^{2}=-\left[U^{T} A U\right]_{0}^{1} .
$$

Since $A$ is symmetric there is an orthonormal matrix $X$ which diagonalizes $A$ as $A=X \Lambda X^{T}$, where $\Lambda=\operatorname{diag}[\bar{u}+\bar{c}, \bar{u}-\bar{c}]$ contains the eigenvalues of $A$. The energy estimate can hence be rewritten as

$$
\|U\|_{t}^{2}=\left[\left(X^{T} U\right)^{T} \Lambda\left(X^{T} U\right)\right]_{0}^{1}
$$

and we can see that one boundary condition is required on each boundary, since by assumption we have $\bar{u}<\bar{c}$. Hence, as $\varepsilon \rightarrow 0$ the number of boundary conditions change from 2 to 1 on the left boundary for the primal problem. For the dual problem, the number of boundary condition change from 2 to 1 on the right boundary. As a consequence it is required that the matrices $H_{L}$ in (12) and $A+H_{R}^{T}$ in (20) both have rank 1 and non-zero top rows, and that energy estimates can be obtained. The choices which fulfills these requirements are given in

Proposition 3.5. Let

$$
\alpha_{L}=\bar{c}-\bar{u}, \quad \beta_{L}=\bar{u}-\bar{c}, \quad \alpha_{R}=\bar{u}-\bar{c}, \quad \beta_{R}=\bar{c}-\bar{u} .
$$

Then the boundary conditions

$$
\begin{aligned}
& H_{L} U-B U_{x}=0, \\
& H_{R} U+B U_{x}=0,
\end{aligned}
$$

constitute a well-posed set of boundary conditions for the incompletely parabolic system of equations (3) and its dual equations (16), and converge to a well-posed set of boundary conditions for the hyperbolic system (23) and its dual as $\varepsilon \rightarrow 0$. 
Proof. On the left boundary it is required that $H_{L}$ has rank 1 and non-zero top row. In this case, two boundary conditions will be imposed if $\varepsilon \neq 0$ and one linearly independent condition if $\varepsilon=0$. On the right boundary it is required that $A+H_{R}^{T}$ has rank 1 and non-zero top row. Thus the dual equations will have two conditions if $\varepsilon \neq 0$ and one linearly independent condition if $\varepsilon=0$. Inserting the values of $\alpha_{L, R}$ and $\beta_{L, R}$ in (24) gives

$$
H_{L}=\left[\begin{array}{cc}
\bar{u} & \bar{u} \\
\bar{c} & \bar{c}
\end{array}\right], \quad A+H_{R}^{T}=\left[\begin{array}{cc}
\bar{u} & \bar{u} \\
\bar{c} & \bar{c}
\end{array}\right]
$$

and we can see that the requirements are fulfilled. The energy estimates of both the primal and dual equations, independently of $\varepsilon$, are of the form

$$
\|\xi\|_{t}^{2}+\left\|B \xi_{x}\right\|^{2}=-\xi^{T} M_{L} \xi-\xi^{T} M_{R} \xi
$$

where $M_{L, R}$ are as before. The $2 \times 2$ matrices $M_{L}$ and $M_{R}$ have the same eigenvalues which are given by

$$
\lambda_{1,2}=\bar{c} \pm \sqrt{\bar{c}^{2}-2 \bar{u}(\bar{c}-\bar{u})} \geq 0,
$$

since $\bar{u}<\bar{c}$ by assumption. Hence energy estimates are obtained for all cases.

Remark 3.3. The choices in (24) make the boundary conditions for both the primal and dual equations relate to the characteristics of $A$ by

$$
\begin{aligned}
H_{L} & =A+H_{R}^{T}=X_{L}^{T} \Lambda^{+} X_{L}, \\
-H_{R} & =A-H_{L}^{T}=X_{R}^{T} \Lambda^{-} X_{R},
\end{aligned}
$$

where $X_{L, R}$ are the normalized eigenvector matrices and

$$
\Lambda^{ \pm}=\frac{\Lambda \pm|\Lambda|}{2}
$$

contains the positive and negative eigenvalues of $A$, respectively. See for example $[18,31]$.

\subsection{Discretization, stability and spatial dual consistency}

To discretize systems of equations, it is convenient to introduce the Kronecker product which is defined for arbitrary matrices $C, D$ as

$$
C \otimes D=\left[\begin{array}{cccc}
C_{11} D & C_{12} D & \cdots & C_{1 n} D \\
C_{21} D & C_{22} D & \cdots & C_{2 n} D \\
\vdots & \ddots & \ddots & \vdots \\
C_{n 1} D & C_{n 2} D & \cdots & C_{n n} D
\end{array}\right]
$$


For the matrix inverse and transpose we have

$$
(C \otimes D)^{-1, T}=C^{-1, T} \otimes D^{-1, T}
$$

if the usual matrix inverses are defined. Furthermore, a useful property which will be extensively used, is the mixed product property,

$$
(C \otimes D)(\tilde{C} \otimes \tilde{D})=C \tilde{C} \otimes D \tilde{D},
$$

if the usual matrix products are defined.

Using the Kronecker product, equation (3) with the boundary conditions (6) can be discretized using the SBP-SAT technique as

$$
\begin{aligned}
\frac{d}{d t} U_{h}+\left(D_{1} \otimes A\right) U_{h} & =\left(D_{2} \otimes B\right) U_{h} \\
& +\left(P^{-1} E_{0} \otimes \Sigma_{L}\right)\left(\left(I_{N+1} \otimes H_{L}\right) U_{h}-\left(D_{1} \otimes B\right) U_{h}-G_{L}\right) \\
& +\left(P^{-1} E_{N} \otimes \Sigma_{R}\right)\left(\left(I_{N+1} \otimes H_{R}\right) U_{h}+\left(D_{1} \otimes B\right) U_{h}-G_{R}\right),
\end{aligned}
$$

where $\Sigma_{L, R}$ are $2 \times 2$ matrices which have to be determined for stability. The second derivative is approximated using the wide operator, $D_{2}=D_{1} D_{1}=\left(P^{-1} Q\right)^{2}$. The matrices $\Sigma_{L, R}$ are given in

Proposition 3.6. The scheme (25) is energy stable by choosing

$$
\Sigma_{L}=\Sigma_{R}=-I
$$

Proof. We let $G_{L}=G_{R}=0$ and apply the energy method to (25). By using the SBP properties of the operators, we obtain

$$
\begin{aligned}
\left\|U_{h}\right\|_{t}^{2}+2\left\|\left(D_{1} \otimes B\right) U_{h}\right\|^{2} & =U_{h}^{T}\left(E_{0} \otimes\left(A+\Sigma_{L} H_{L}+H_{L}^{T} \Sigma_{L}^{T}\right)\right) U_{h} \\
& -U_{h}^{T}\left(E_{N} \otimes\left(A-\Sigma_{R} H_{R}-H_{R}^{T} \Sigma_{R}^{T}\right)\right) U_{h} \\
& -2 U_{h}^{T}\left(E_{0} D_{1} \otimes\left(B+\Sigma_{L} B\right)\right) U_{h} \\
& +2 U_{h}^{T}\left(E_{N} D_{1} \otimes\left(B+\Sigma_{R} B\right)\right) U_{h} .
\end{aligned}
$$

By choosing $\Sigma_{L}=\Sigma_{R}=-I$, equation (27) simplifies to

$$
\begin{aligned}
\left\|U_{h}\right\|_{t}^{2}+2\left\|\left(D_{1} \otimes B\right) U_{h}\right\|^{2}= & -U_{h}^{T}\left(E_{0} \otimes\left(-A+H_{L}+H_{L}^{T}\right)\right) U_{h} \\
& -U_{h}^{T}\left(E_{N} \otimes\left(A+H_{R}+H_{R}^{T}\right)\right) U_{h},
\end{aligned}
$$

where, by construction in the continuous case,

$$
-A+H_{L}+H_{L}^{T} \geq 0, \quad A+H_{R}+H_{R}^{T} \geq 0
$$


according to propositions 3.1 and 3.3. Since the Kronecker product preserves positive (semi) definiteness, we have

$$
\begin{aligned}
\left\|U_{h}\right\|_{t}^{2}+2\left\|\left(D_{1} \otimes B\right) U_{h}\right\|^{2}= & -U_{h}^{T}\left(E_{0} \otimes\left(-A+H_{L}+H_{L}^{T}\right)\right) U_{h} \\
& -U_{h}^{T}\left(E_{N} \otimes\left(A+H_{R}+H_{R}^{T}\right)\right) U_{h} \leq 0
\end{aligned}
$$

and the scheme is energy stable.

The choices of penalty matrices in (26) renders the scheme not only energy stable, but also spatially dual consistent. To prove this we must show that the discrete adjoint operator $L_{h}^{*}$ consistently approximates the continuous adjoint $L^{*}$, including the dual boundary conditions (10) and (19). This is done in

Proposition 3.7. The scheme (25) is spatially dual consistent with the choices of $\Sigma_{L, R}$ given in (26).

Proof. For $G_{L, R}=0$, we can write the scheme (25) as

$$
\frac{d}{d t} U_{h}+L_{h} U_{h}=0
$$

where

$$
\begin{aligned}
L_{h} & =\left(D_{1} \otimes A\right)-\left(D_{2} \otimes B\right) \\
& +\left(P^{-1} E_{0} \otimes I_{2}\right)\left(\left(I_{N+1} \otimes H_{L}\right)-\left(D_{1} \otimes B\right)\right) \\
& +\left(P^{-1} E_{N} \otimes I_{2}\right)\left(\left(I_{N+1} \otimes H_{R}\right)+\left(D_{1} \otimes B\right)\right) .
\end{aligned}
$$

The discrete dual operator is defined by

$$
L_{h}^{*}=\left(P \otimes I_{2}\right)^{-1} L_{h}^{T}\left(P \otimes I_{2}\right)
$$

and a straight forward calculation shows that

$$
\begin{aligned}
L_{h}^{*} & =-\left(D_{1} \otimes A\right)-\left(D_{2} \otimes B\right) \\
& -\left(P^{-1} E_{0} \otimes I_{2}\right)\left(\left(I_{N+1} \otimes\left(A-H_{L}^{T}\right)\right)+\left(D_{1} \otimes B\right)\right) \\
& +\left(P^{-1} E_{N} \otimes I_{2}\right)\left(\left(I_{N+1} \otimes\left(A+H_{R}^{T}\right)\right)+\left(D_{1} \otimes B\right)\right)
\end{aligned}
$$

which is a consistent approximation of (7) including the dual boundary conditions (10) and (19). The scheme is hence spatially dual consistent.

Not only is the scheme dual consistent, the discrete dual scheme is also stable which is shown in 
Proposition 3.8. The discrete dual scheme

$$
\frac{d}{d \tau} \theta_{h}+L_{h}^{*} \theta_{h}=0
$$

is stable with $L_{h}^{*}$ given in (29).

Proof. The energy estimate of (30) is given by

$$
\begin{aligned}
\left\|\theta_{h}\right\|_{t}^{2}+2\left\|\left(D_{1} \otimes B\right) \theta_{h}\right\|^{2}= & -\theta_{h}^{T}\left(E_{0} \otimes\left(-A+H_{L}+H_{L}^{T}\right)\right) \theta_{h} \\
& -\theta_{h}^{T}\left(E_{N} \otimes\left(A+H_{R}+H_{R}^{T}\right)\right) \theta_{h} \leq 0,
\end{aligned}
$$

which is again stable by construction of the continuous boundary conditions. The discretization of the primal problem (28) is hence simultaneously a stable discretization of the dual problem (30).

Remark 3.4. To obtain a stable and dual consistent scheme with the flux based boundary conditions, only one penalty parameter at each boundary is required. From the stability analysis both of them are determined uniquely as the identity matrix, which is also sufficient for spatial dual consistency. For Dirichlet boundary conditions, nine non-trivial penalty parameters were required, and the stability requirements were not sufficient for dual consistency.

\section{Convergence and errors}

A forcing function has been chosen such that an analytical solution is known, and the rates of convergence and errors are computed with respect to the analytical

solution. This is known as the method of manufactured solutions. The solution in this case is given by

$$
\begin{aligned}
& p(x, t)=(\arctan (x)-\delta \cos (\alpha x-t)+1) e^{-x^{2}}, \\
& u(x, t)=(\arctan (x)+\delta \sin (\alpha x-t)+1) e^{-x^{2}},
\end{aligned}
$$

and the functionals by

$$
\begin{aligned}
& J(p)=1+\frac{\pi}{4}-\frac{\log (2)}{2}+\frac{\delta(\sin (t-\alpha)-\sin (t))}{\alpha}, \\
& J(u)=1+\frac{\pi}{4}-\frac{\log (2)}{2}+\frac{\delta(\cos (t)-\cos (t-\alpha))}{\alpha} .
\end{aligned}
$$

Typical values of the parameters are

$$
\bar{u}=0.5, \quad \bar{c}=1, \quad \varepsilon=10^{-2}, \quad \delta=0.1, \quad \alpha=5 \pi .
$$




\begin{tabular}{|c|c|c|c|c||c|c|c|c|}
\hline & \multicolumn{7}{|c||}{ Flux 1 } & \multicolumn{5}{c|}{ Dirichlet } \\
\hline$N$ & 64 & 96 & 128 & 160 & 64 & 96 & 128 & 160 \\
\hline \multicolumn{8}{|c|}{ 3rd-order } \\
\hline$p$ & 3.0530 & 3.0426 & 3.0377 & 3.0345 & 3.5301 & 3.3552 & 3.2357 & 3.1667 \\
$u$ & 2.8870 & 2.9740 & 2.9973 & 3.0061 & 3.6191 & 3.5355 & 3.4484 & 3.3812 \\
$J(p)$ & 2.5584 & 3.9209 & 4.2617 & 4.4285 & 3.9162 & 4.1626 & 4.2958 & 4.3643 \\
$J(u)$ & 4.2536 & 4.2841 & 4.3698 & 4.4192 & 3.7781 & 3.5831 & 4.2249 & 4.5709 \\
\hline \multicolumn{7}{|c|}{ 4th-order } \\
\hline$p$ & 3.9728 & 4.2644 & 4.3975 & 4.4581 & 3.6293 & 4.3273 & 4.4387 & 4.4715 \\
$u$ & 5.0736 & 4.6958 & 4.5338 & 4.4499 & 4.6610 & 5.0024 & 4.9220 & 4.7676 \\
$J(p)$ & 2.2407 & 4.5614 & 5.5758 & 5.9743 & 4.0073 & 5.1505 & 5.4943 & 5.6757 \\
$J(u)$ & 4.4065 & 5.6051 & 6.0071 & 6.2345 & 3.9695 & 4.3917 & 5.2755 & 5.5775 \\
\hline \multicolumn{7}{|c|}{5 th-order } \\
\hline$p$ & 5.4375 & 5.2515 & 5.0597 & 4.9655 & 5.2057 & 5.7541 & 5.7028 & 5.5475 \\
$u$ & 4.1043 & 5.0985 & 5.2485 & 5.2911 & 5.7376 & 5.1068 & 4.8237 & 4.8244 \\
$J(p)$ & 4.9722 & 7.4842 & 7.8269 & 8.1507 & 6.0856 & 7.1729 & 7.7521 & 8.2018 \\
$J(u)$ & 6.4334 & 7.1878 & 7.7660 & 8.1503 & 5.9507 & 6.9309 & 7.7971 & 8.2529 \\
\hline
\end{tabular}

Table 1: Convergence rates for the variables and functionals using both flux based and Dirichlet boundary conditions

In Table 1 we present the numerical results regarding the order of convergence of the solution and functionals using both flux based and Dirichlet boundary conditions. The time integration is performed until time $t=0.2$ with the classical 4th-order Runge-Kutta method using 1000 time steps. The convergence rates for the flux based boundary conditions (6) are not sensitive to the choice of the continuous parameters, and in the numerical examples that follow, we have chosen the marginal values

$$
\alpha_{L}=\bar{c}, \quad \beta_{L}=\bar{u} / 2, \quad \alpha_{R}=-\bar{c}, \quad \beta_{R}=-\bar{u} / 2 .
$$

The choices in (24) give very similar results and are excluded.

As can be seen from Table 1, both schemes results in superconvergent functionals with similar rates of convergence. The error in the solutions and functionals as a function of time for 3rd-order and $N=32$ grid points can be seen in Figure 1 and 2, respectively. We denote the values in (31) by "Flux 1" and the characteristic values in (24) by "Flux 2".

As we can see from Figure 1 and 2, in particular the characteristic flux based scheme (Flux 2) has significantly smaller functional errors.

In $[21,1]$ it was shown that schemes based on characteristic boundary conditions for hyperbolic problems, have non-growing errors in time - so called error bounded 


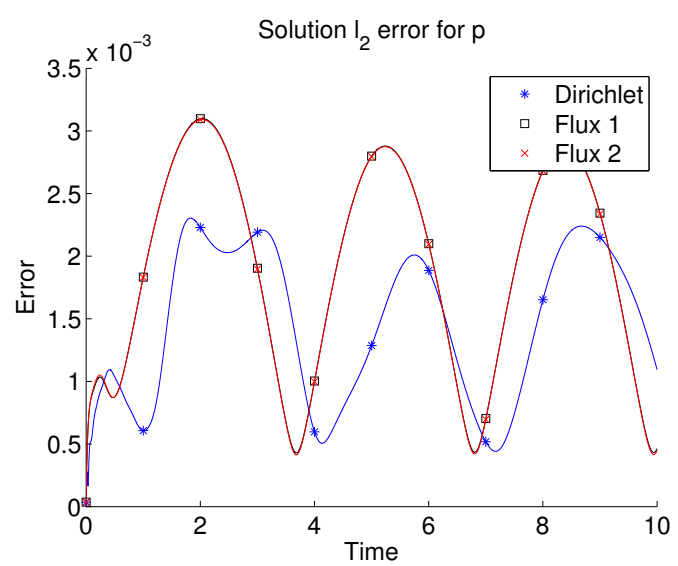

(a) Solution $l_{2}$ error for $p$

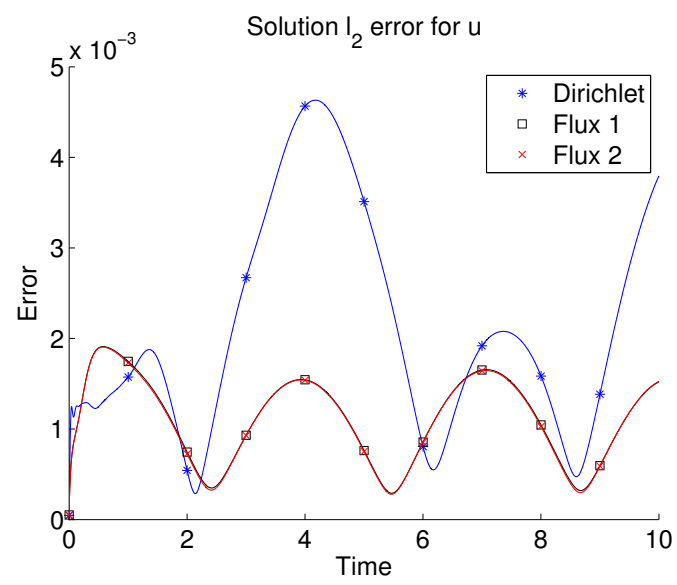

(b) Solution $l_{2}$ error for $u$

Figure 1: Solution $l_{2}$ errors as a function of time using $N=32$ grid points and 3rd-order for both the Dirichlet and the flux based boundary conditions

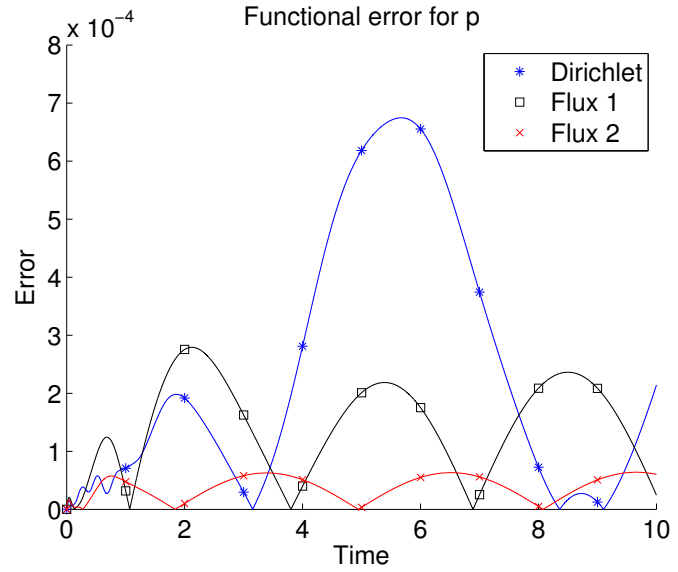

(a) Functional error for $J(p)$

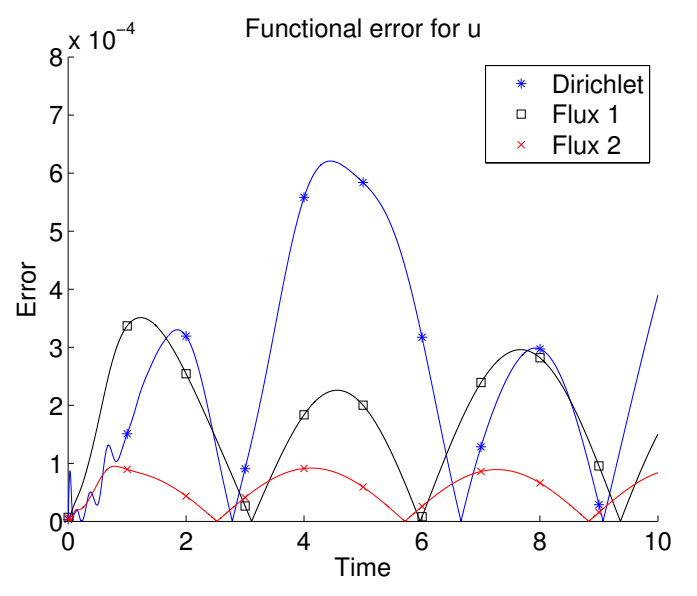

(b) Functional error for $J(u)$

Figure 2: Functional errors as a function of time using $N=32$ grid points and 3rd-order for both the Dirichlet and the flux based boundary conditions 


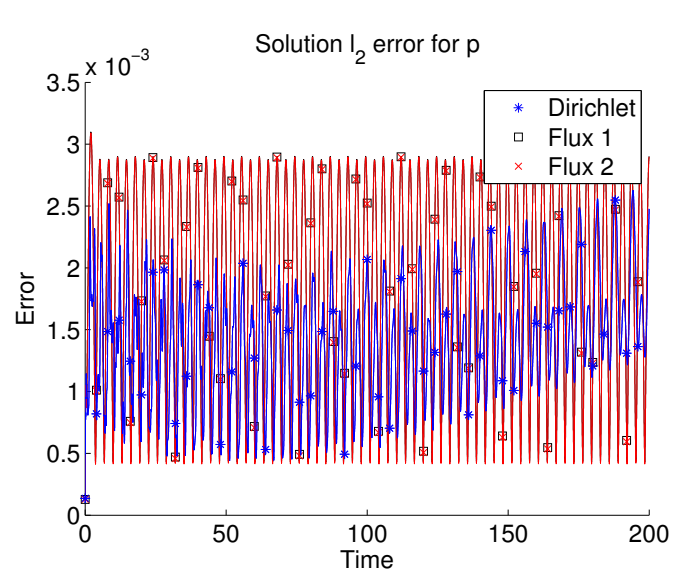

(a) Solution $l_{2}$ error for $p$

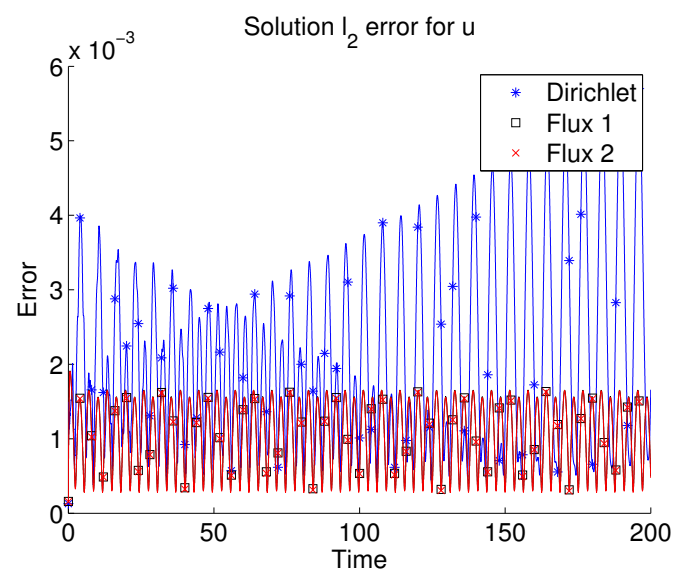

(b) Solution $l_{2}$ error for $u$

Figure 3: Solution $l_{2}$ errors as a function of time using $N=32$ grid points and 3rd-order for both the Dirichlet and the flux based boundary conditions using $\varepsilon=10^{-6}$

schemes. We can hence expect the discretization with Dirichlet boundary condition to have linearly growing errors in time for $\varepsilon<<1$, while the flux based boundary conditions are error bounded. Indeed, in Figures 3 and 4 we plot the error as a function of time for large times using $\varepsilon=10^{-6}$. The Dirichlet boundary conditions give linearly growing errors while the flux based boundary conditions are error bounded.

With increasing $\varepsilon$, the discretization using the Dirichlet boundary conditions also becomes error bounded due to the parabolic effects. Since the characteristic choices in (24) converges uniformly to well-posed and stable boundary conditions for the hyperbolic system, we have an error bounded scheme for all values of $\varepsilon$, including $\varepsilon=0$.

\section{Spectral analysis}

A consistent numerical scheme should have eigenvalues which converge to the continuous eigenvalues as the mesh is refined $[23,3]$. The continuous spectrum of a PDE is obtained by Laplace transforms in time to reduce the PDE to an ordinary differential equation, and solve the corresponding Sturm-Liouville problem. Let

$$
\hat{U}=\int_{0}^{\infty} e^{s t} U d t
$$




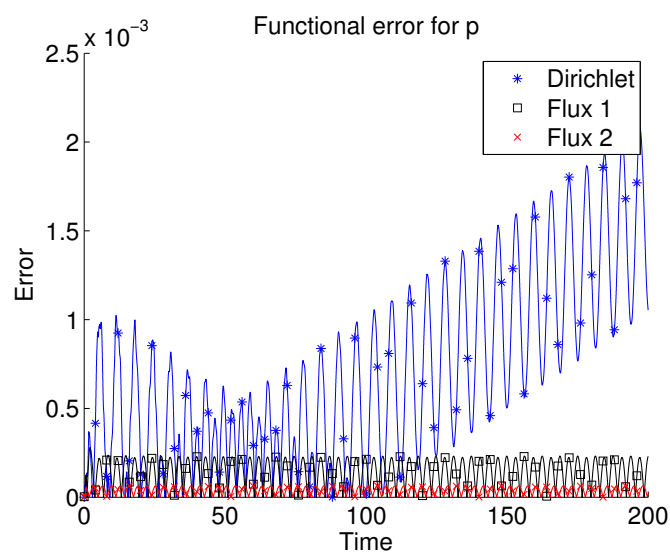

(a) Functional error for $J(p)$

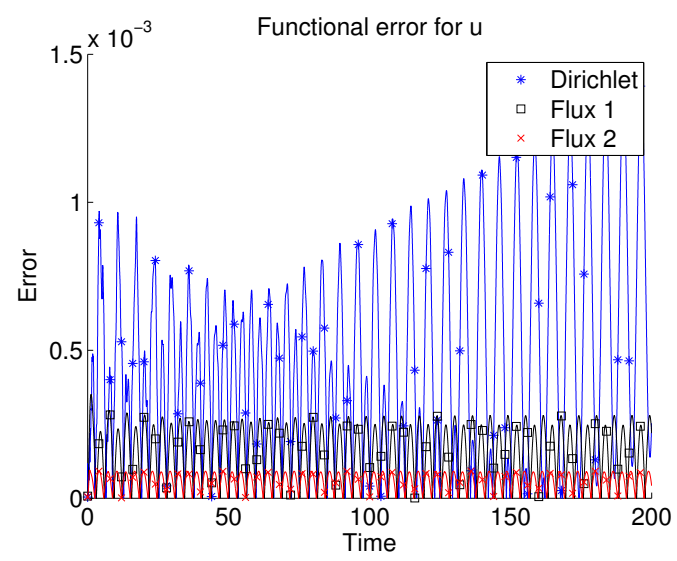

(b) Functional error for $J(u)$

Figure 4: Functional errors as a function of time using $N=32$ grid points and 3rd-order for both the Dirichlet and the flux based boundary conditions using $\varepsilon=10^{-6}$

denote the Laplace transform of $U$. By ignoring the initial condition, as usual, and Laplace transforming (3), we obtain

$$
s \hat{U}-A \hat{U}_{x}=B \hat{U}_{x x}
$$

and the ansatz $\hat{U}=e^{k x} \Psi$ turns (32) into the eigenvalue problem

$$
\left(s I+k A-k^{2} B\right) \Psi=0 .
$$

The general solution to (32), assuming distinct roots, can be written as

$$
\hat{U}=\sum_{i=1}^{3} \sigma_{i} e^{k_{i}} \Psi_{i},
$$

where $k_{i}$ are the roots of the polynomial $\operatorname{det}\left(s I+k A-k^{2} B\right)$ and $\Psi_{i}=\left[\Psi_{i}(1), \Psi_{i}(2)\right]^{T}$ are the corresponding eigenvectors. Once the $k_{i}$ and $\Psi_{i}$ have been determined, the system of equations for determining $\sigma_{i}$ can be written as

$$
E(s) \sigma=0
$$

after the homogeneous boundary conditions have been applied. The continuous spectrum is then given by the values of $s$ such that $\operatorname{det}(E(s))=0$. See [11] for 
further details on the proceedure. For the Dirichlet boundary conditions we obtain the $3 \times 3$ matrix

$$
E_{D}(s)=\left[\begin{array}{ccc}
\Psi_{1}(1) & \Psi_{2}(1) & \Psi_{3}(1) \\
\Psi_{1}(2) & \Psi_{2}(2) & \Psi_{3}(2) \\
e^{k_{1}} \Psi_{1}(2) & e^{k_{2}} \Psi_{2}(2) & e^{k_{3}} \Psi_{3}(2)
\end{array}\right]
$$

The flux based boundary conditions yield the $4 \times 3$ matrix

$$
E_{F}(s)=\left[\begin{array}{ccc}
\left(H_{L}-k_{1} B\right) \Psi_{1} & \left(H_{L}-k_{2} B\right) \Psi_{2} & \left(H_{L}-k_{3} B\right) \Psi_{3} \\
\left(H_{R}+k_{1} B\right) e^{k_{1}} \Psi_{1} & \left(H_{R}+k_{2} B\right) e^{k_{2}} \Psi_{2} & \left(H_{R}+k_{3} B\right) e^{k_{3}} \Psi_{3}
\end{array}\right],
$$

where the 3 rd row is zero and hence $E_{F}(s)$ is condensed to a $3 \times 3$ matrix. Analytical solutions to $\operatorname{det}(E(s))=0$ can in general not be obtained due to the algebraic complexity. For scalar equations, analytical results are available in [3], while for more complicated equations, numerical methods have to be used. See [23] for more details.

Once $E_{D, F}(s)$ has been computed, eigenvalues $s$ from the discrete spectrum can be inserted into the matrices to see whether or not a discrete eigenvalue actually belongs to the spectrum of the PDE. This technique can be used to verify convergence of discrete eigenvalues with certain properties. The semi-discretization of a linear system of equations can be written as

$$
\frac{d}{d t} u_{h}=K u_{h}+f
$$

where the entire spatial discretization, including the boundary conditions, are included in the matrix $K$. The discrete spectrum can be modified and tuned for certain purposes depending on the boundary treatment.

The maximum real part and absolute value of the spectra are important since the first determines the convergence rate to steady-state $[18,23]$ while the second is a measure of the stiffness of the system. Both of these depend on the value of $\varepsilon$, which can be viewed as the viscosity. To see the effects, we show the maximum real part and absolute value in Table 2. For small values of $\varepsilon$, the characteristic flux based conditions, Flux 2, has significantly smaller real part of the spectrum. The difference is about a factor of 50-100 compared to Flux 1, and between 3 and 8 compared to Dirichlet. The spectrum of Flux 1 can, however, easily be shifted to the left in the complex plane by having strict inequalities in (24). The maximum absolute values are similar for small values of $\varepsilon$, while larger values forces the flux based conditions towards a much stiffer discretization. 


\begin{tabular}{|c|c|c|c||c|c|c|}
\hline & \multicolumn{3}{|c||}{ Maximum real part } & \multicolumn{3}{c|}{ Maximum absolute value } \\
\hline$\varepsilon$ & Dirichlet & Flux 1 & Flux 2 & Dirichlet & Flux 1 & Flux 2 \\
\hline $10^{-6}$ & -0.412 & -0.029 & -1.515 & 34.4 & 34.4 & 32.1 \\
$10^{-5}$ & -0.412 & -0.029 & -1.517 & 34.4 & 34.4 & 32.1 \\
$10^{-4}$ & -0.412 & -0.029 & -1.539 & 34.4 & 34.4 & 32.1 \\
$10^{-3}$ & -0.412 & -0.029 & -1.753 & 34.4 & 34.5 & 32.1 \\
0.01 & -0.415 & -0.029 & -3.158 & 34.5 & 34.9 & 32.1 \\
0.1 & -0.463 & -0.030 & -1.492 & 35.7 & 85.0 & 121.0 \\
1 & -0.537 & -0.027 & -0.498 & 45.6 & 961.3 & 987.0 \\
\hline
\end{tabular}

Table 2: Maximum real part and absolute values of the spectras using 3rd order and $N=16$

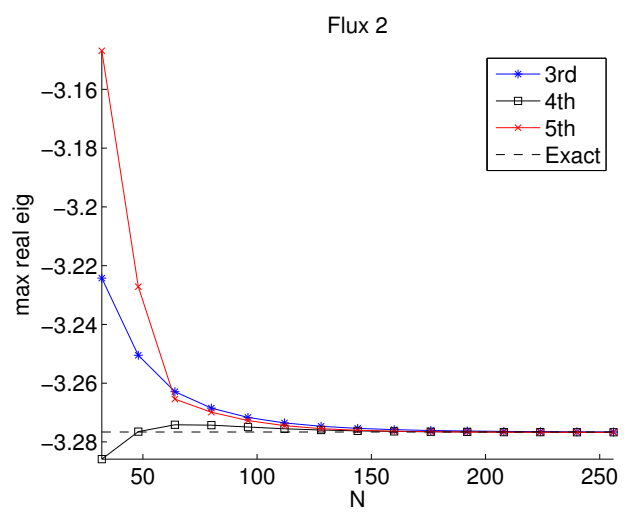

(a) Maximum real part, all orders of accuracy

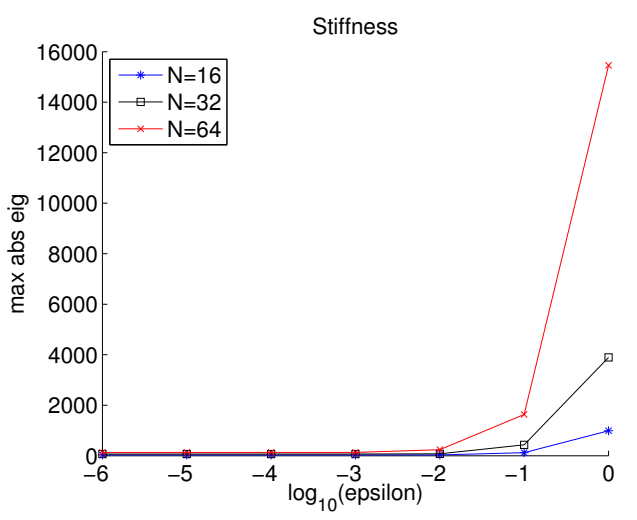

(b) Maximum absolute value, 3rd-order

Figure 5: Convergence of maximum real part and absolute value for the Flux 2 boundary conditions

The continuous eigenvalue with real part closest to zero can be computed from the determinants of $E_{D, F}(s)$. As an example, we show the convergence of Flux 2 towards this eigenvalue in Figure 5(a). In Figure 5(b) we show the increase in stiffness for different values of $\varepsilon$ as the mesh is refined.

\section{Conclusions}

New flux based boundary conditions for a linear incompletely parabolic system of equations have been derived. The boundary conditions are constructed such that both the primal and dual problems are well-posed. Depending on parameter variations in the new boundary conditions, choices can be made to either provide wellposedness independently of sub- or supersonic conditions, or such that convergence to the hyperbolic system is ensured for the subsonic case. 
The numerical scheme based on the new boundary conditions can be constructed to be both energy stable and dual consistent. Compared to a discretization using standard Dirichlet boundary conditions, the new scheme is significantly simpler since reduction to first order form, and additional penalty parameters, can be avoided.

The solutions and functionals computed using the flux based boundary conditions are more accurate and have better damping properties. Long time computations showed that the new scheme can provide error boundedness independently of the amount of viscosity, even in the hyperbolic limit.

Eigenvalue computations showed that the maximum real part of the discrete spectrum converges to the analytical value. The flux based boundary conditions can provide smaller real parts than a discretization with Dirichlet boundary conditions which is beneficial for steady-state computations. The stiffness is, however, increased for large viscosity.

The analysis of this model problem will be applied to the more complicated compressible Navier-Stokes equations in future work.

\section{Acknowledgments}

This work has partly been carried out within the IDIHOM project which is supported by the European Commission under contract No. ACP0-GA-2010-265780.

\section{References}

[1] S. Abarbanel, A. Ditkowski, and B. Gustafsson. On error bounds of finite difference approximations to partial differential equations - temporal behavior and rate of convergence. Journal of Scientific Computing, 15:79-116, 2000.

[2] S. Abarbanel and D. Gottlieb. Optimal time splitting for two- and threedimensional Navier-Stokes equations with mixed derivatives. Journal of Computational Physics, 41(1):1-33, 1981.

[3] J. Berg and J. Nordström. Spectral analysis of the continuous and discretized heat and advection equation on single and multiple domains. Applied Numerical Mathematics, 62(11):1620-1638, 2012.

[4] J. Berg and J. Nordström. Superconvergent functional output for timedependent problems using finite differences on summation-by-parts form. Journal of Computational Physics, 231(20):6846-6860, 2012. 
[5] M. H. Carpenter, J. Nordström, and D. Gottlieb. A stable and conservative interface treatment of arbitrary spatial accuracy. Journal of Computational Physics, 148(2):341-365, 1999.

[6] K. J. Fidkowski and D. L. Darmofal. Review of output-based error estimation and mesh adaptation in computational fluid dynamics. AIAA Journal, 49(4):673-694, 2011.

[7] M. B. Giles, M. G. Larson, J. M. Levenstam, and E. Süli. Adaptive error control for finite element approximations of the lift and drag coefficients in viscous flow. Technical report, Report NA-97/06, Oxford University Computing Laboratory, 1997.

[8] M. B. Giles and N. A. Pierce. An introduction to the adjoint approach to design. Flow, Turbulence and Combustion, 65:393-415, 2000.

[9] M. B. Giles and N. A. Pierce. Superconvergent lift estimates through adjoint error analysis. Innovative Methods for Numerical Solutions of Partial Differential Equations, 2001.

[10] M. B. Giles and E. Süli. Adjoint methods for PDEs: A posteriori error analysis and postprocessing by duality. Acta Numerica, 11:145-236, 2002.

[11] B. Gustafsson, H.-O. Kreiss, and J. Oliger. Time Dependent Problems and Difference Methods. Wiley Interscience, 1995.

[12] J. E. Hicken. Output error estimation for summation-by-parts finite-difference schemes. Journal of Computational Physics, 231(9):3828-3848, 2012.

[13] J. E. Hicken and D. W. Zingg. Superconvergent functional estimates from summation-by-parts finite-difference discretizations. SIAM Journal on Scientific Computing, 33(2):893-922, 2011.

[14] J. E. Hicken and D. W. Zingg. Summation-by-parts operators and high-order quadrature. Journal of Computational and Applied Mathematics, 237(1):111$125,2013$.

[15] A. Jameson. Aerodynamic design via control theory. Journal of Scientific Computing, 3:233-260, September 1988.

[16] K. Mattsson. Summation by parts operators for finite difference approximations of second-derivatives with variable coefficients. Journal of Scientific Computing, pages 1-33, 2011. 
[17] K. Mattsson and J. Nordström. Summation by parts operators for finite difference approximations of second derivatives. Journal of Computational Physics, $199(2): 503-540,2004$.

[18] J. Nordström. The influence of open boundary conditions on the convergence to steady state for the Navier-Stokes equations. Journal of Computational Physics, 85(1):210-244, 1989.

[19] J. Nordström. The use of characteristic boundary conditions for the NavierStokes equations. Computers \& Fluids, 24(5):609-623, 1995.

[20] J. Nordström. Conservative finite difference formulations, variable coefficients, energy estimates and artificial dissipation. Journal of Scientific Computing, $29: 375-404,2006$.

[21] J. Nordström. Error bounded schemes for time-dependent hyperbolic problems. SIAM Journal on Scientific Computing, 30(1):46-59, 2008.

[22] J. Nordström and M. H. Carpenter. High-order finite difference methods, multidimensional linear problems, and curvilinear coordinates. Journal of Computational Physics, 173(1):149-174, 2001.

[23] J. Nordström, S. Eriksson, and P. Eliasson. Weak and strong wall boundary procedures and convergence to steady-state of the Navier-Stokes equations. Journal of Computational Physics, 231(14):4867-4884, 2012.

[24] J. Nordström and M. Svärd. Well-posed boundary conditions for the NavierStokes equations. SIAM Journal on Numerical Analysis, 43(3):1231-1255, 2005.

[25] N. A. Pierce and M. B. Giles. Adjoint recovery of superconvergent functionals from PDE approximations. SIAM Review, 42(2):247-264, 2000.

[26] N. A. Pierce and M. B. Giles. Adjoint and defect error bounding and correction for functional estimates. Journal of Computational Physics, 200:769-794, November 2004.

[27] O. Pironneau. On optimum design in fluid mechanics. Journal of Fluid Mechanics, 64(01):97-110, 1974.

[28] B. Strand. Summation by parts for finite difference approximations for d/dx. Journal of Computational Physics, 110(1):47-67, 1994. 
[29] J. C. Strikwerda. Initial boundary value problems for incompletely parabolic systems. Communications on Pure and Applied Mathematics, 30(6):797-822, 1977.

[30] M. Svärd. On coordinate transformations for summation-by-parts operators. Journal of Scientific Computing, 20:29-42, 2004.

[31] M. Svärd, M. H. Carpenter, and J. Nordström. A stable high-order finite difference scheme for the compressible Navier-Stokes equations, far-field boundary conditions. Journal of Computational Physics, 225(1):1020-1038, 2007.

[32] M. Svärd, K. Mattsson, and J. Nordström. Steady-state computations using summation-by-parts operators. Journal of Scientific Computing, 24(1):79-95, 2005.

[33] M. Svärd and J. Nordström. On the order of accuracy for difference approximations of initial-boundary value problems. Journal of Computational Physics, 218(1):333-352, 2006.

[34] D. A. Venditti and D. L. Darmofal. Adjoint error estimation and grid adaptation for functional outputs: Application to quasi-one-dimensional flow. Journal of Computational Physics, 164(1):204-227, 2000.

[35] D. A. Venditti and D. L. Darmofal. Anisotropic grid adaptation for functional outputs: application to two-dimensional viscous flows. Journal of Computational Physics, 187(1):22-46, 2003. 